\title{
A SELF-DUAL SEPTIMIC POSSESSING SEVEN OF EACH KIND OF THE SIMPLE SINGULARITIES, AND AUTOPOLAR BY SEVEN RECTANGULAR HYPERBOLAS AND A CIRCLE*
}

\author{
BY D. C. DUNCAN
}

The equation of the locus in homogeneous rectangular coordinates, $x, y, z$, is developed under the assumptions:

i. The seven cusps are distributed at equal intervals about the unit-circle, their cuspidal tangents concurrent at $(0,0,1)$.

ii. The curve is invariant under rotations about the origin through angles $2 \pi k / 7,(k=1,2,3, \cdots, 6)$.

iii. Each cuspidal tangent is an axis of symmetry.

As an immediate consequence of ii and iii, one node and one point of inflexion must lie on each cuspidal tangent.

The most general equation of a locus of the seventh order is

$$
\begin{aligned}
A f_{7}(x, y)+ & B z f_{6}(x, y)+C z^{2} f_{5}(x, y)+D z^{3} f_{4}(x, y) \\
& +E z^{4} f_{3}(x, y)+F z^{5} f_{2}(x, y)+G z^{6} f_{1}(x, y)+H z^{7}=0 .
\end{aligned}
$$

The various $f_{i}(x, y)$ are homogeneous polynomial functions of degree $i$ in $x, y$, which must be individually absolutely invariant under the rotations about the origin through angles $2 k \pi / 7$. Now $f_{i}(x, y)=0$ represents $i$ straight lines through the origin. But it is manifestly impossible for $i$ straight lines to be invariant under rotations of $2 \pi / 7$ if $i<7$, unless they are the isotropic lines $\left(x^{2}+y^{2}\right)^{h}=0$. Therefore we may write $f_{6}(x, y) \equiv\left(x^{2}+y^{2}\right)^{3}$, $f_{4}(x, y) \equiv\left(x^{2}+y^{2}\right)^{2}, f_{2}(x, y) \equiv\left(x^{2}+y^{2}\right), C=0, E=0, F=0$, since the equation is to be real and to represent a real locus. Accordingly in polar coordinates the locus is represented by

$$
A \rho^{7} \cos (7 \theta+\alpha)+B \rho^{6}+D \rho^{4}+F \rho^{2}+H=0 .
$$

Placing a cusp at $\rho=1, \theta=0$, causes $\alpha$ to vanish.

When $\theta=0$, the roots of $A \rho^{7}+B \rho^{6}+D \rho^{4}+F \rho^{2}+H=0$ must include a triple root for $\rho=1$, the cusp, and a double root for $\rho=a$, the node. It is next shown that for just one essential set of real values for $A, B, D, F, H$, the equation can have such roots. Assume

\footnotetext{
* Presented to the Society, March 18, 1933.
} 
$A \rho^{7}+B \rho^{6}+D \rho^{4}+F \rho^{2}+H \equiv A(\rho-1)^{3}(\rho-a)^{2}\left(\rho^{2}-p \rho+q\right)$.

Since the coefficients of $\rho^{5}, \rho^{3}, \rho$ are zero, one has necessarily

$$
\begin{array}{rlr}
a^{2}+6 a+3+p(2 a+3)+q & =0, \\
3 a^{2}+2 a+p\left(3 a^{2}+6 a+1\right)+q\left(a^{2}+6 a+3\right) & =0, \\
+p a^{2} & +q\left(3 a^{2}+2 a\right) & =0 .
\end{array}
$$

The consistency of these equations requires the vanishing of the determinant, that is,

$$
\left|\begin{array}{ccc}
a^{2}+6 a+3 & 2 a+3 & 1 \\
3 a^{2}+2 a & 3 a^{2}+6 a+1 & a^{2}+6 a+3 \\
0 & a^{2} & 3 a^{2}+2 a
\end{array}\right|=0 \text {. }
$$

Expanding and factoring, one obtains

$$
8 a(a+1)^{2}\left(3 a^{3}+12 a^{2}+16 a+4\right)=0 .
$$

The vanishing of $a$ would imply, contrary to hypothesis, a multiple point at the origin; the vanishing of $(a+1)$ is also to be discarded as the node cannot also lie on the unit circle for this would imply a circle meeting a septimic in 7 cusps and 7 nodes or 28 points, which is impossible. Hence the node is to be sought in the cubic factor. Fortunately this is reducible and yields the single root, $-\left\{(2 / 3)(7 / 2)^{1 / 3}+(4 / 3)+(7 / 6)(2 / 7)^{1 / 3}\right\}$. Moreover

$$
p=-\frac{(3 a+2)\left(a^{2}+6 a+3\right)}{6(a+1)^{2}}, \quad q=\frac{a\left(a^{2}+6 a+3\right)}{6(a+1)^{2}},
$$

whence it is found that $p^{2}-4 q$ is definitely negative, indicating no other real intersection of the septimic with the $x$-axis.

Accordingly, for one single essential set of real values of $A, B, D, F, H$, the equation $A \rho^{7} \cos 7 \theta+B \rho^{6}+D \rho^{4}+F \rho^{2}+H=0$ represents a locus having the singularities situated according to the initial hypotheses. The nodes are found to be crunodes. It is clearly impossible for such a locus to consist of curves of lower order; hence the locus must be non-degenerate. Its equation in homogeneous rectangular coordinates is 


$$
\begin{aligned}
& \left(5(7 / 2)^{1 / 3}+10+14(2 / 7)^{1 / 3}\right)\left(x^{7}-21 x^{5} y^{2}+35 x^{3} y^{4}-7 x y^{6}\right) \\
& \quad+7\left((13 / 3)(7 / 2)^{1 / 3}+71 / 12+(28 / 3)(2 / 7)^{1 / 3}\right)\left(x^{2}+y^{2}\right)^{3} z \\
& \quad-21\left((43 / 8)(7 / 2)^{1 / 3}+8+(49 / 4)(2 / 7)^{1 / 3}\right)\left(x^{2}+y^{2}\right)^{2} z^{3} \\
& \quad+7\left((67 / 4)(7 / 2)^{1 / 3}+101 / 4+(77 / 2)(2 / 7)^{1 / 3}\right)\left(x^{2}+y^{2}\right) z^{5} \\
& \quad-\left((953 / 24)(7 / 2)^{1 / 3}+361 / 6+(1099 / 12)(2 / 7)^{1 / 3}\right) z^{7}=0 .
\end{aligned}
$$

Now if a polarity interchanges the cusps and inflexional tangents, cuspidal tangents and points of inflexion, the polarity will convert the entire locus into itself. This fact is due to the unique existence of the locus with the cusps in the specified position; the polar reciprocal curve possessing the same cusps must be coincident with the original septimic. The polarities are now exhibited.

The point and line coordinates of the following singularities are immediately deducible.

$$
\begin{aligned}
& \begin{array}{llll}
x & : & y & : z
\end{array} \\
& \text { Cusps } \quad \cos (2 k \pi / 7): \sin (2 k \pi / 7): 1 \text {, } \\
& \text { Pts. inflex. } \cos (2 k \pi / 7): \sin (2 k \pi / 7): 0 \text {, } \\
& u \quad: \quad v \quad: w \\
& \left(c=-\frac{52(7 / 2)^{1 / 3}+71+112(2 / 7)^{1 / 3}}{12\left[5(7 / 2)^{1 / 3}+10+14(2 / 7)^{1 / 3}\right]}\right),(k=0,1,2, \cdots, 6) \text {. }
\end{aligned}
$$

It is observed that only the following eight polarities interchange these singular elements:

$$
\left\{\begin{aligned}
\rho u & =x, \\
\rho v & =y, \\
\rho w & =c z,
\end{aligned}\right\} \begin{aligned}
\rho u & =x \cos (2 k \pi / 7)-y \sin (2 k \pi / 7), \\
\rho v & =-x \sin (2 k \pi / 7)-y \cos (2 k \pi / 7), \\
\rho w & =c z, \quad(k=0,1,2, \cdots, 6) .
\end{aligned}
$$

Therefore the septimic is invariant under eight polarities, the first by the real circle $x^{2}+y^{2}+c z^{2}=0$, the others by the seven rectangular hyperbolas which touch this circle and whose principal axes coincide with the cuspidal tangents respectively. On account of the symmetry of the locus it is also invariant under six 
correlations which are the products of the polarities $(k=1,2,3$, $4,5,6)$ with reflexion about any cuspidal tangent.

\section{Correlations (Fourteen)}

$$
\begin{array}{cccc}
u & : & v & : w \\
x & : & y & : c z \\
x \cos (2 k \pi / 7)-y \sin (2 k \pi / 7):-x \sin (2 k \pi / 7) & -y \cos (2 k \pi / 7): c z, \\
& & (k=0,1, \cdots, 6) .
\end{array}
$$

Polarities.

$x \cos (2 k \pi / 7)+y \sin (2 k \pi / 7):-x \sin (2 k \pi / 7)+y \cos (2 k \pi / 7): c z$, $(k=1,2, \cdots, 6)$.

$$
\begin{aligned}
& \text { Collineations (Fourteen) } \\
\rho x= & x \cos (2 k \pi / 7) \mp y \sin (2 k \pi / 7), \\
\rho y= & x \sin (2 k \pi / 7) \pm y \cos (2 k \pi / 7), \\
\rho z= & z, \quad(k=0,1,2, \cdots, 6) .
\end{aligned}
$$

The equations of the double tangents are now immediately obtainable by polarizing the nodes. They are

$$
\begin{aligned}
& \left\{(2 / 3)(7 / 2)^{1 / 3}+4 / 3+(7 / 6)(2 / 7)^{1 / 3}\right\} x \cos (2 k \pi / 7)+y \sin (2 k \pi / 7) \\
& +\frac{52(7 / 2)^{1 / 3}+71+112(2 / 7)^{1 / 3}}{12\left\{5(7 / 2)^{1 / 3}+10+14(2 / 7)^{1 / 3}\right\}} z=0,(k=0,1,2, \cdots, 6) .
\end{aligned}
$$

The line equation of the septimic is immediately obtained by replacing $x, y, z$ by $u, v, w / c$.

The form of the curve is very approximately realized by drawing secant lines through alternate points of the seven points equally distributed about the unit circle, and then removing the chords from the interior of the circle. It is unipartite and of deficiency 1 .

The University of California 